\title{
Peritoneal Dialysis or Haemodialysis for Kidney Failure?
}

\author{
Badri Man Shrestha' \\ 'Department of General Surgery, Sheffield Teaching Hospitals NHS Trust, Sheffield, UK.
}

Although kidney transplantation (KT) is the best form of treatment for kidney failure patients, more that $75 \%$ of patients do need dialysis in the form of peritoneal dialysis (PD) or haemodialysis (HD) during their life-time for several reasons, which include a, non-availability of a kidney donor, unsuitability for KT, failed KT and unexpected diagnosis of kidney failure. The choice between PD and HD has remained a subject of debate. However, the balance is swinging in favour of PD because of several advantages associated with the utilization of PD in comparison to HD.

PD confers better quality of life compared to HD due to autonomy, flexibility, avoidance of regular hospital visits for the patients and their relatives, better cosmesis related to HD vascular access sites and freedom of pain from repeated cannulation for HD. PD preserves the residual renal function and vascular access sites for the future dialysis. Analysis of European Renal Association and European Dialysis and Transplantation Association Registry data of 198076 dialysis patients followed over a period of 20 years has shown progressive increase in 5-year survival of patients on PD compared to those on HD. ${ }^{1}$ There is also significant economic advantage of PD over HD globally. In the United Kingdom, the annual cost of PD per patient is $£ 17500$ in comparison to $£ 35000$ for HD. A recent meta-analysis has shown that pre-KT PD is associated with better post-transplant patient survival than pre-KT HD. The incidence of delayed graft function after KT is significantly low in patients on PD compared to those on HD. These results suggest the PD may be the preferred dialysis modality for patients expected to receive a $\mathrm{KT} .^{2}$

Despite significant advantages of PD over HD, PD remains an underutilised modality of treatment for kidney failure all over the world. Hong Kong has the highest utilisation of PD in the world, where over $75 \%$ of the patients are on PD, whereas in majority of European countries and in the United States of America (USA), the PD utilisation rate is under $15 \%$. The "PD first" philosophy of the health care system has led to exemplary success of PD in Hong Kong, where every patient with kidney failure is offered PD. HD is provided only to those patients who are considered unsuitable for PD. Patients, who are suitable for PD but opt for HD, must pay the cost of HD personally. ${ }^{3}$ In several countries, the choice of HD is guided by the financial remuneration system, which is particularly so in the private hospital settings.

The reason for underutilisation PD has not been fully explored. However, two studies published from the USA have shown that there is significant deficiency in the exposure on the management of PD patients to the nephrology and surgical trainees in their residency programme, which could be the case in most of the countries in the world. Nephrologists, in the fore front of the management of the kidney failure patients, should be able to offer unbiased advice with regards to the advantages and disadvantages of PD vs. HD and allow the patients to make a choice of dialysis regimen independently. ${ }^{4,5}$ Several surveys have shown that over $50 \%$ of kidney failure patients opt for PD when an unbiased advice is offered to them by the nephrologists. ${ }^{6}$

Correspondence: Dr. BM Shrestha, Department of General Surgery, Northern General Hospital, Sheffield S5 7AU, UK. Email: shresthabm@doctors.net.uk, Fax: +441142714604. 
The success of PD depends upon the presence of a functioning PD catheter without any associated complications, which is dependent on the surgical technique of PD catheter insertion and the skills of the surgeons. Of the various techniques of $P D$ catheter insertion, including open surgical, blind percutaneous, fluoroscopic-guided percutaneous, basic and advanced laparoscopic insertion techniques; the advanced laparoscopic insertion technique comprising of laparoscopic rectus sheath tunneling, omentopexy, adhesiolysis, colopexy, salpingectomy and epiploectomy is becoming increasingly popular because of its highest success rate. ${ }^{7}$
The advantages of PD over HD have been well recognised. It is imperative that the institutions providing care to the kidney failure patients should revisit their dialysis patients and programmes and follow a pathway that is in the best interest of their kidney failure patients. Expansion of existing PD programme through training and education of the patients, medical and surgical team, development of local clinical practice guidelines, maintenance of a registry, regular audit of clinical outcomes and integration of the available resources will help towards effective delivery of PD service. ${ }^{8}$

\section{ACKNOWLEDGEMENTS}

Author would like to acknowledge graphic designer Mr. OM Prakash Yadav for his support.

\section{Conflict of Interest: None.}

\section{REFERENCES}

1. van de Luijtgaarden MW, Jager KJ, Segelmark M, et al. Trends in dialysis modality choice and related patient survival in the ERA-EDTA Registry over a 20-year period. Nephrol Dial Transplant. 2016;31(1):120-8. [PubMed | DOI]

2. Joachim E, Gardezi AI, Chan MR, Shin JI, Astor BC, Waheed S. Association of Pre-Transplant Dialysis Modality and Post-Transplant Outcomes: A Meta-Analysis. Perit Dial Int. 2017;37(3):259-65. [PubMed | DOI]

3. Kwong VW, Li PK. Peritoneal Dialysis in Asia. Kidney Dis (Basel). 2015;1(3):147-56. [PubMed | DOI]

4. Mehrotra R. Peritoneal dialysis education: Challenges and innovation. Semin Dial. 2018 Mar;31(2):107-10. [uuMed | DOI]

5. Wong LP, Liebman SE, Wakefield KA, Messing S. Training of surgeons in peritoneal dialysis catheter placement in the United States: a national survey. Clin J Am Soc Nephrol. 2010;5(8):1439-46. [PubMed | DOI]
6. Korevaar JC, Feith GW, Dekker FW, van Manen JG, Boeschoten EW, Bossuyt PM, et al. Effect of starting with hemodialysis compared with peritoneal dialysis in patients new on dialysis treatment: a randomized controlled trial. Kidney Int. 2003;64(6):2222-8. [ubMed | DOI]

7. Shrestha B, Shrestha D, Kumar A, Shrestha A, Boyes S, Wilkie M. Advanced laparoscopic insertion of peritoneal dialysis catheter: systematic review and meta-analysis. Peritoneal Dialysis International [Internet]. [cited 2018 Jan2]. Available from:https://www.researchgate. net/publication/321947394_Advanced_laparoscopic_ peritoneal_dialysis_catheter_insertion_Systematic_review_ and_meta-analysis 2018. [․ㅏll Text]

8. Wilkie M, Jenkins S, Shrestha B. The UK Renal Association Guidelines - Peritoneal [Internet]. [cited 2018 Jan 15]. Available from: https://renal.org/wp-content/ uploads/2017/06/ peritoneal-access-5th-edition-1.pdf. [피l Text] 